\title{
IncRNA CASC2/miR-18a-5p axis regulates the malignant potential of nasopharyngeal carcinoma by targeting RBBP8
}

\author{
WEN-JIE MIAO, DONG-JIE YUAN, GUO-ZHENG ZHANG, QI LIU, HUI-MIN MA and QIAN-QIAN JIN
}

\author{
Department of Otolaryngology, The First Affiliated Hospital of Xinxiang Medical University, \\ Weihui, Henan 453100, P.R. China
}

Received August 15, 2018; Accepted November 23, 2018

DOI: $10.3892 /$ or.2018.6941

\begin{abstract}
Nasopharyngeal carcinoma (NPC) is a prevalent head and neck tumor which has a high mortality rate in Southeast Asia, especially in Southern China. Cancer susceptibility candidate 2 (CASC2) is a newly identified long non-coding RNA (lncRNA) that has been found to play a suppressive role in several types of tumors. However, the expression and functional role of CASC2 in NPC are still unclear. In the present study, using NPC tissues, cells and transplanted mice, we investigated the mechanism of CASC2-mediated regulation of NPC. We showed that the CASC2 level is reduced in NPC tissues and cells. CASC2 downregulation promoted proliferation and inhibited apoptotic cell death in NPC cells. In contrast, CASC2 upregulation inhibited proliferation and increased apoptosis. There were putative binding sites of microRNA (miR)-18a-5p in the promoter of CASC2. The level of miR-18a-5p was upregulated in NPC tissues and cells. We further confirmed that CASC 2 could directly bind with miR-18a-5p and inhibit miR-18a-5p expression, using reporter gene and RNA immunoprecipitation assays. miR-18a-5p suppressed CASC2 upregulation-mediated decrease in proliferation and increase in apoptotic cell death. Bioinformatics predicted the putative binding site of miR-18a-5p in the $3^{\prime}$ untranslated region of C-terminal binding protein interacting protein (CtIP)/RBBP8. It was further confirmed that miR-18a-5p could directly bind with RBBP8 and inhibit RBBP8 expression. Downregulation of RBBP8 inhibited the anti-miR-18a-5p-mediated increase in apoptosis and decrease in proliferation. Downregulation of CASC2 increased tumor growth, increased the level of miR-18a-5p and decreased RBBP8 expression in vivo. In summary, CASC2 regulates NPC malignancy through modulation of RBBP8 via sponging miR-18a-5p. Our findings highlight the CASC2/miR-18a-5p/RBBP8 axis in NPC pathogenesis and
\end{abstract}

Correspondence to: Professor Wen-Jie Miao, Department of Otolaryngology, The First Affiliated Hospital of Xinxiang Medical University, 88 Jiankang Road, Weihui, Henan 453100, P.R. China E-mail: wenjiemiao2018@126.com

Key words: nasopharyngeal carcinoma, lncRNA CASC2, miR-18a-5p, RBBP8, proliferation provide new biomarkers and potential targets for the therapy of NPC.

\section{Introduction}

Nasopharyngeal carcinoma (NPC) is a prevalent head and neck tumor which has a mortality rate of $15-50$ per 100,000 individuals in Southeast Asia, especially in the southern area of China $(1,2)$. Globally, there are 80,000 new cases of NPC diagnosed each year and approximately 50,000 patients succumb to this neoplasm annually (3). A variety of factors, including viral infection, genetics and environment, contribute to the occurrence of NPC (4). NPC can be divided into three histologic subtypes based on the World Health Organization (WHO) guidelines: Type I, keratinizing squamous cell carcinoma; type II, non-keratinizing differentiated carcinoma; and type III, nonkeratinizing undifferentiated carcinoma (5). It is proposed that NPC tumorigenesis and progression is a multistep process which involves multiple genetic and epigenetic changes $(6,7)$. Although the outcome of NPC treatment has been improved by advanced radiotherapy and chemotherapy, the 5-year survival rate of NPC patients is still at 50-70\% (8) and NPC therapy is mainly limited by tumor recurrence and distant metastasis $(9,10)$. Therefore, it is of great importance to identify novel biomarkers and new targets for the therapy of NPC.

Long non-coding RNAs (lncRNAs) are a family of non-coding RNAs (ncRNAs) that have less than 200 bases in length and possess limited ability for protein-coding (11-14). Numerous studies have confirmed that dysregulation of lncRNA expression is associated with the development of a number of tumors (15-17). It is well established that lncRNAs play important roles in the regulation of a battery of cellular and biological processes, including development, differentiation, de-differentiation, proliferation, autophagy and apoptosis (18). Cancer susceptibility candidate 2 (CASC2), a newly identified lncRNA which is located on chromosome 10q2, was found to play a tumor-suppressive role in some types of tumors such as hepatocellular carcinoma, bladder, breast cancer, osteosarcoma, gastric cancer, lung adenocarcinoma and endometrial cancer (19-25). MicroRNAs (miRNAs), a set of RNAs which are 19-22 nt in length with no coding ability, function as key regulators in tumorigenesis and progression (26). Recently, it has been proposed that one of the functions of lncRNAs is to sponge specific miRNAs and thus to affect the mRNA 
expression of target genes, the effect of which is believed to play a role in the tumorigenic process $(27,28)$.

Bioinformatic software predicted that there were binding sites of miR-18a-5p in the $3^{\prime}$ untranslated region (UTR) of CASC2. However, the expression and functional role of CASC2 and miR-18a-5p in the pathogenesis of NPC remain unclear. The goal of the present study was to identify the pattern of CASC 2 and miR-18a-5p expression in NPC, and to evaluate the role of CASC 2 and miR-18a-5p interaction in the regulation of NPC cell proliferation and apoptosis. In the present study, we found a reduction in CASC2 expression and an increase in miR-18a-5p expression in NPC tissues and cells. We further revealed the critical roles of CASC2 and miR-18a-5p in the modulation of the apoptosis and proliferation of NPC cells. We identified that CASC2 inhibited the tumorigenesis and malignant potential of NPC via targeting the $\mathrm{miR}-18 \mathrm{a}-5 \mathrm{p} / \mathrm{C}$-terminal binding protein interacting protein (CtIP)/RBBP8 axis.

\section{Materials and methods}

Ethics statement. The present study was conducted according to the principles expressed in the Declaration of Helsinki, and the use of clinical sample tissues was approved by the Ethics Committee of the First Affiliated Hospital of Xinxiang Medical University (Weihui, China). Written informed consent was obtained from all the enrolled patients.

Tissue samples and cells. Twenty-five NPC patients (age, 25-72 years; 14 female and 11 male patients) who underwent surgery at the First Affiliated Hospital of Xinxiang Medical University (from November 3, 2014 to August 23, 2015) were included in the study. NPC tissues and normal nasopharyngeal tissues were obtained from the patients. All patients had received no therapy prior to biopsy. All histologic diagnoses were conducted by the two independent pathologist. Informed consent was received from all the patients.

Normal nasopharyngeal epithelial cells (N69) and three NPC cell lines (SUNE1, SUNE2 and 6-10B) were purchased from the American Type Culture Collection (ATCC; Manassas, VA, USA). 293T cells were purchased from the Shanghai Institutes for Biological Sciences Cell Resource Centre (Shanghai, China). The cell lines were incubated in RPMI-1640 medium (Gibco; Thermo Fisher Scientific, Inc., Waltham, MA, USA) supplemented with $10 \%$ fetal bovine serum (FBS; Gibco, Thermo Fisher Scientific, Inc.) in a humidified atmosphere at $37^{\circ} \mathrm{C}$ with $5 \% \mathrm{CO}_{2}$.

Transfection of plasmids. PCR was performed to amplify the CASC2 full length sequence and then the pcDNA3.1 vector (Thermo Fisher Scientific, Inc.) was used to establish the pcDNA-CASC2 overexpression plasmid (CASC2). The siRNAs [si-CASC2 and its negative control (si-NC)], microRNA inhibitors [anti-miR-18a-5p and its negative control (anti-miR-NC)] and microRNA mimics [miR-18a-5p and its negative control (miR-NC)] were obtained from Shanghai GenePharma Co., Ltd. (Shanghai, China). The transfection of oligonucleotides and plasmids was performed using Lipofectamine ${ }^{\mathrm{TM}} 2,000$ reagent (Invitrogen; Thermo Fisher Scientific, Inc.) according to the manufacturer's instructions.
Evaluation of cell proliferation. Proliferation was evaluated using Cell Counting Kit-8 (CCK-8; Beyotime Institute of Biotechnology, Haimen, China) assay kit. After the treatment, medium was removed and fresh medium was added followed by the addition of $10 \mu \mathrm{l}$ of CCK- 8 solution. Then, the cells were incubated at $37^{\circ} \mathrm{C}$ for $2 \mathrm{~h}$. Finally, the absorbance at $450 \mathrm{~nm}$ was assessed using a Bio-Rad iMark microplate absorbance reader (Bio-Rad Laboratories Inc., Hercules, CA, USA). CCK-8 assay was performed in triplicate experiments.

Apoptosis. TUNEL assay kits (Roche Diagnostics, Basel, Switzerland) were used to detect apoptotic cell death in SUNE1 and $6-10 \mathrm{~B}$ cells. In brief, cells were trypsinized and resuspended at the concentration of $1 \times 10^{6}$ cells $/ \mathrm{ml} 48 \mathrm{~h}$ after transfection. The apoptotic rate of NPC cells was analyzed using a flow cytometer (FACSCalibur; BD Biosciences, Franklin Lakes, NJ, USA) and quantified by CellQuest software (BD Biosciences). Relative apoptosis was expressed as a percentage of apoptotic cells.

Luciferase assay. PCR was performed to amplify the partial sequences of CASC2 which contained the putative binding sites of miR-18a-5p. The sequences were then cloned into the pmirGLO Dual-Luciferase miRNA Target Expression Vector (Promega Corp.,Fitchburg, WI, USA). GeneArt ${ }^{\mathrm{TM}}$ Site-Directed Mutagenesis system (Thermo Fisher Scientific, Inc.) was used to induce site-directed mutagenesis of miR-18a-5p complementary bases in the sequences of CASC2. Then, 293T cells were transfected with the constructed wild-type (WT) and mutant (MUT) reporter vectors, respectively, in the presence of anti-miR-18a-5p or anti-miR-NC and miR-18a-5p mimics or miR-NC. The activity of luciferase was measured using the Dual-Luciferase Assay system (Promega) and then normalized to Renilla luciferase activity based on the manufacturer's instructions. The assay was performed in triplicate experiments.

$R N A$ extraction, reverse transcription and $R T-q P C R$. Total RNA was extracted from cells and tissues using TRIzol reagent (Life Technologies; Thermo Fisher Scientific, Inc.) following the manufacturer's instructions. After that, the quality of RNA was assessed using the NanoDrop 1,000 (NanoDrop Technologies; Thermo Fisher Scientific, Inc.). For the detection of mRNA and lncRNA expression, real-time PCR (RT-qPCR) reaction was conducted using SYBR-Green (Takara Biotechnology, Co., Lt., Dalian, China). The amplification was carried out on an ABI 7,500 Real-Time PCR system (Applied Biosystems; Thermo Fisher Scientific, Inc.). GAPDH and $\beta$-actin were regarded as the housekeeping genes for the normalization of target genes. For the measurement of miRNA, synthesis of cDNA was carried out using the qScript microRNA cDNA Synthesis kit (Quantabio, Beverly, MA, USA) following the manufacturer's protocols. MicroRNA RT-qPCR was performed using a miScript SYBR-Green PCR kit (Qiagen, Hilden, Germany) as per the manufacturer's instructions. U6 was regarded as the housekeeping gene for the normalization of target miRNAs. $2^{-\Delta \Delta C q}$ method was used to evaluate the relative fold change of gene expression (29).

RNA immunoprecipitation assay. RNA immunoprecipitation (RIP) assay was performed to detect the interaction between 
A

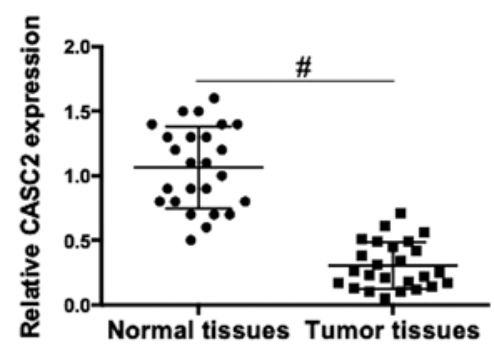

C

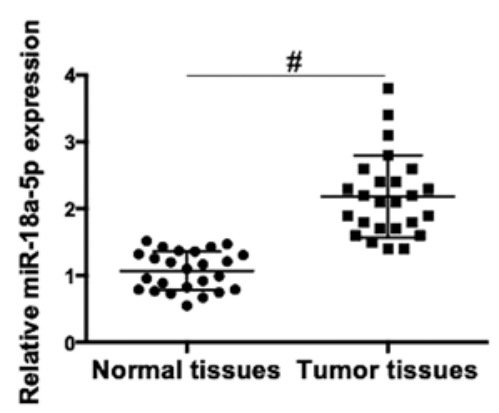

B

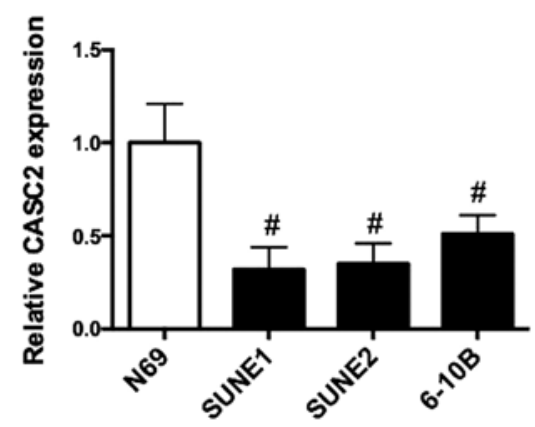

D

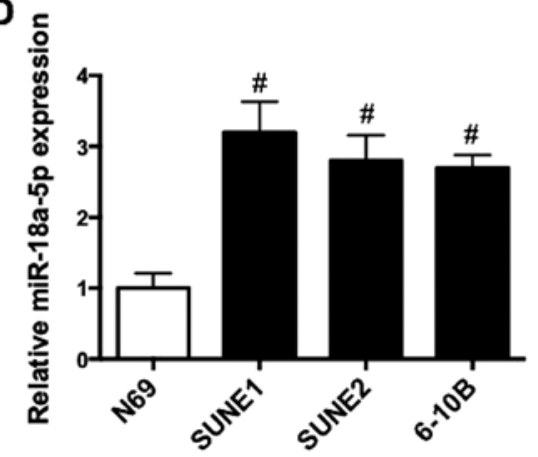

Figure 1. CASC2 expression is reduced and miR-18a-5p expression is increased in NPC tissues and cells. (A) mRNA expression of CASC2 in normal nasopharyngeal tissues $(\mathrm{n}=25)$ and NPC tissues $(\mathrm{n}=25)$ was determined using RT-qPCR. (B) mRNA expression of CASC2 in normal nasopharyngeal epithelial N69 cells $(n=69)$ and in NPC cell lines (SUNE1, SUNE2 and 6-10B) was determined using RT-qPCR. (C) mRNA expression of miR-18a-5p in NPC tissues (n=25) and normal nasopharyngeal tissues ( $\mathrm{n}=25$ ) was determined using RT-qPCR. (D) miR-18a-5p mRNA expression in NPC cell lines (SUNE1, SUNE2 and 6-10B) and normal nasopharyngeal epithelial cells (N69) was determined. " $\mathrm{P}<0.05$ compared with the respective control. CASC2, cancer susceptibility candidate 2 ; NPC, nasopharyngeal carcinoma.

IncRNA and miRNA, using an Imprint RNA immunoprecipitation kit (Sigma-Aldrich; Merck KGaA, Darmstadt, Germany). In brief, anti-IgG (negative control) and anti-Argomaute2 (anti-Ago2) were added to the cell lysate and incubated overnight at $4^{\circ} \mathrm{C}$. After that, Protein A magnetic beads were added to the mixture to form a complex of immunoprecipitation. After that, extra protein and DNA were removed to purify the complex. Finally, the enrichment of CASC2 and miR-18a-5p in the immunoprecipitated complex was evaluated by RT-qPCR assay.

Lentivirus production and infection. The CASC2-shRNA lentivirus vector (LV-shCASC2) was constructed by Shanghai GenePharma Biotech Co., Ltd. LV-shCASC2 or empty vector (LV-shNC) lentiviruses were used to transfect SUNE1 cells. Then, the transfected cells were purified with $5 \mu \mathrm{g} / \mathrm{ml}$ puromycin to obtain cells with stable CASC2 knockdown.

Xenograft mouse model. The animal experiment was carried out following the Guidelines for the Care and Use of Laboratory Animals of the National Institutes of Health. The study was approved by the Ethics Committee of the First Affiliated Hospital of Xinxiang Medical University. Twelve male BALB/c nude mice (18-22 g, 6-8 weeks old) were purchased from the Animal Center of of Xinxiang Medical University. The mice were housed in a specific pathogen free (SPF) animal laboratory under temperature $\left(23 \pm 2^{\circ} \mathrm{C}\right)$ and humidity $(55 \pm 5 \%)$ condition with a standard light cycle (12 h light/dark) and free access to food and water. To establish the xenograft model, $8 \times 10^{6} \mathrm{SUNE} 1$ cells with or without stable knockdown of CASC2 were subcutaneously inoculated into the mice. The experimental period was 6 weeks. Tumor volume was measured using a caliper and calculated according to the following formula: volume $=0.5 \mathrm{x}$ length $\mathrm{x}$ width $\mathrm{x}$ width . After the animal experiment, mice were euthanized using isoflurane and tumor tissues were excised for the evaluation of weight and then stored at $-80^{\circ} \mathrm{C}$ for further analysis.

Statistical analysis. All data are expressed as mean \pm SD. Differences between groups were analyzed with one-way analysis of variance (ANOVA) followed by Tukey's test or Student's t- test. P-values $<0.05$ were considered to be statistically significant, and all analyses were two-sided. All statistical analyses were performed using GraphPad Prism 6.1 software (GraphPad Software Inc., San Diego, CA, USA).

\section{Results}

CASC2 expression is downregulated and miR-18a-5p expression is upregulated in NPC tissues and cell lines. The mRNA expression patterns of CASC2 and miR-18a-5p in NPC tissues and cells were firstly assessed. We showed that the expression of CASC2 was significantly reduced in NPC tissues compared with that in normal nasopharyngeal tissues (Fig. 1A). Similarly, CASC2 expression was significantly decreased in NPC cell lines compared with that in epithelial N69 cells (Fig. 1B). In contrast with the pattern of CASC2 expression, the level of miR-18a-5p in NPC tissues was significantly higher than 

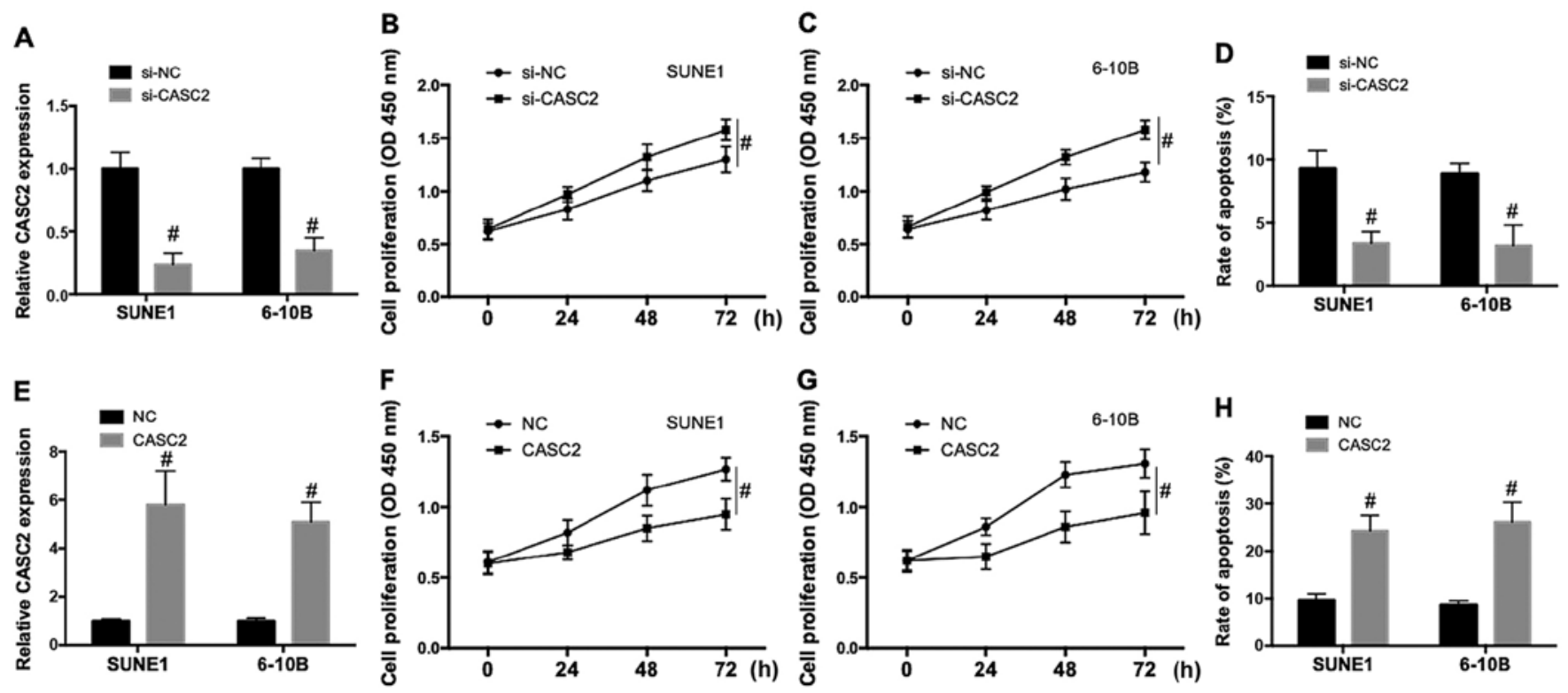

Figure 2. CASC2 knockdown promotes proliferation and inhibits apoptosis in NPC cells. (A) mRNA expression of CASC2 in NPC cells transfected with si-NC or si-CASC2 was determined using RT-qPCR. (B and C) Cell proliferation of NPC cells transfected with si-NC or si-CASC2 was determined using the CCK-8 assay. (D) Apoptosis of NPC cells transfected with si-NC or si-CASC2 was determined using TUNEL assay. (E) mRNA expression of CASC2 was determined using RT-qPCR in pcDNA or pcDNA-CASC2-transfected NPC cells. (F and G) Cell proliferation of NPC cells transfected with pcDNA or pcDNA-CASC2 was determined using the CCK-8 assay. (H) Apoptosis of NPC cells transfected with pcDNA or pcDNA-CASC 2 was determined using TUNEL assay. ${ }^{\#} \mathrm{P}<0.05$ compared with the respective control. CASC2, cancer susceptibility candidate 2; NPC, nasopharyngeal carcinoma; CCK-8, Cell Counting Kit-8.

that in normal nasopharyngeal tissues (Fig. 1C). Additionally, miR-18a-5p expression was significantly increased in NPC cell lines compared with that in epithelial N69 cells (Fig. 1D). The findings indicated that dysregulation of CASC 2 and miR-18a-5p expression may be involved in the tumorigenesis of NPC.

CASC2 downregulation promotes proliferation and inhibits apoptosis in NPC cells. In order to explore the role of CASC2 in the regulation of NPC malignant potential, SUNE1 and 6-10B cells were transfected with small interference RNA of CASC2 (si-CASC2) or a plasmid carrying the CASC2 sequence to downregulate or upregulate CASC2 expression. The efficiency of the knockdown and overexpression was examined. As shown in Fig. 2A and E, the CASC2 expression level was significantly decreased by si-CASC2 and increased by the plasmid carrying the CASC2 sequence in SUNE1 and 6-10B cells. Knockdown of CASC2 significantly increased cell proliferation in SUNE1 (Fig. 2B) and 6-10B (Fig. 2C) cells and inhibited spontaneous apoptosis in NPC cells (Fig. 2D). Upregulation of CASC2 significantly decreased cell proliferation in SUNE1 (Fig. 2F) and 6-10B cells (Fig. 2G) cells and increased apoptosis in NPC cells (Fig. 2H). The data indicated that CASC2 downregulation promoted proliferation and inhibited apoptotic cell death, while CASC2 upregulation decreased proliferation and promoted apoptosis in NPC cells.

CASC2 directly inhibits miR-18a-5p expression. In order to examine the possible mechanism underlying the antitumor role of CASC2 in NPC, bioinformatic analysis was employed to explore CASC2-associated miRNAs. We showed that CASC2 contained putative binding sites for miR-18a-5p (Fig. 3A), indicating that miR-18a-5p may interact with CASC2. This assumption was validated using Dual-Luciferase reporter assay. 293T cells were co-transfected with the constructed wild-type (WT) and mutant (MUT) CASC2 luciferase vectors, anti-miR-NC or anti-miR-18a-5p and miR-NC or miR-18a-5p mimics. The results also showed that miR-18a-5p mimics significantly reduced the luciferase activities of WT-CASC2, while miR-18a-5p inhibitors increased WT-CASC2 luciferase activities (Fig. 3B). In contrast, miR-18a-5p mimics and miR-18a-5p inhibitors did not significantly affect MUT-CASC2 luciferase activities (Fig. 3B). Considering the important role of Ago2 in the formation of RNA-induced silencing complex and the maturation of miRNAs, we performed RIP assay to measure whether there was endogenous interaction between CASC2 and miR-18a-5p. As evidenced in Fig. 3C, CASC2 and miR-18a-5p expression were significantly increased in the Ago 2 antibody complex compared with that in the IgG antibody complex in SUNE1 cells. In order to further evaluate the role of CASC2 in the regulation of miR-18a-5p, SUNE1 cells were transfected with CASC2-overexpression plasmid or si-CASC2 (Fig. 3D). Upregulation of CASC2 significantly decreased miR-18a-5p expression in SUNE1 cells, while CASC2 knockdown significantly promoted miR-18a-5p expression (Fig. 3E). These data demonstrated that CASC2 may act as a sponge for miR-18a-5p in the NPC cells.

To further explore the role of miR-18a-5p in CASC2-induced regulation of proliferation and apoptosis, SUNE1 and 6-10B cells were co-transfected with CASC2-overexpression plasmid and miR-18a-5p mimics. We revealed that CASC2 overexpression-induced reduction of proliferation was significantly suppressed by miR-18a-5p mimics (Fig. 4A and B). In addition, CASC2 overexpression-induced increase of apoptosis was significantly blocked by miR-18a-5p mimics (Fig. 4C and D). 
A

\section{CASC2WT}

miR-18a-5p

CASC2 MUT
5'-tgaataaCAaTAaATGCACCTTa-3' $\||\||||\|\mid\|$ 3'-gatagacGTgATcTACGTGGAAt-5'

5'-tgaataaUCaGCaCGAUCUUGGa-3'
B

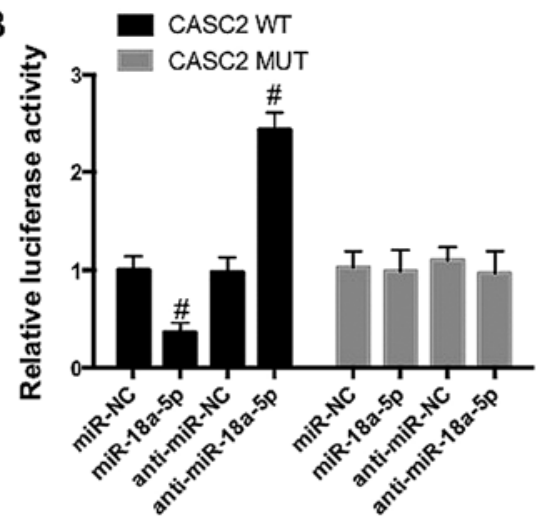

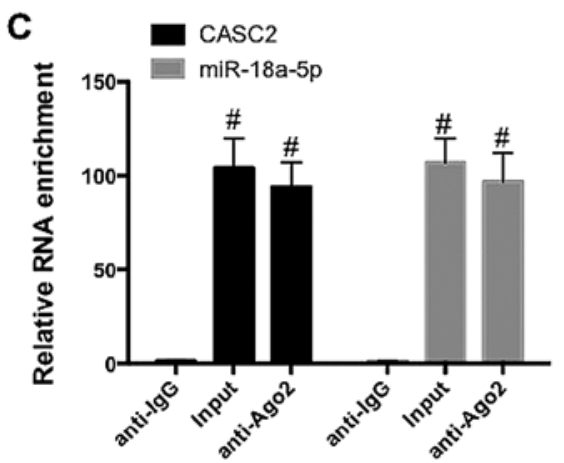
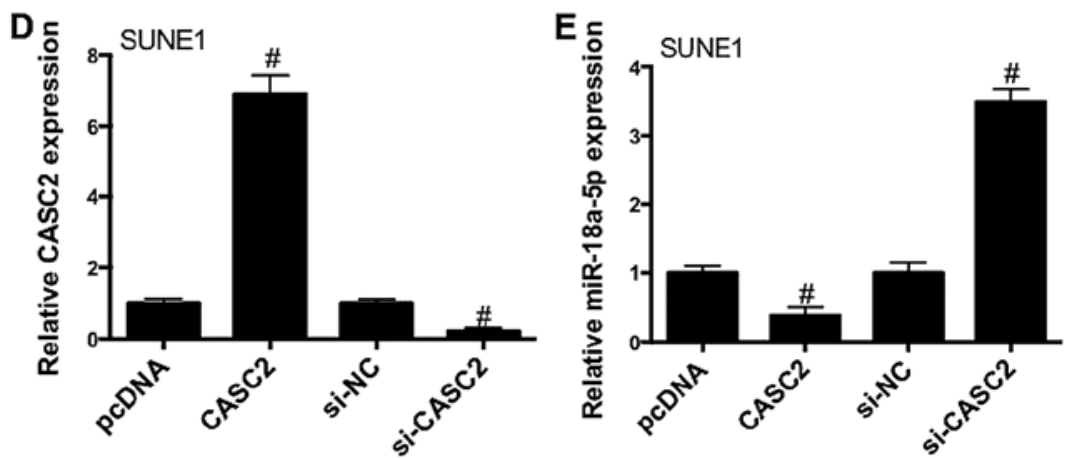

Figure 3. CASC2 suppresses miR-18a-5p expression by direct interaction. (A) Bioinformatic analysis was performed to identify putative binding sites between CASC and miR-18a-5p. (B) 293T cells were co-transfected with WT or MUT CASC2 luciferase vectors and anti-miR-NC or anti-miR-18a-5p and miR-NC or miR-18a-5p, and then the direct interaction between CASC2 and miR-18a-5p was assessed by reporter gene assay. (C) Cell lysate of SUNE1 cells was incubated with anti-Ago2 antibody or anti-IgG antibody to purify RNA complex and RT-qPCR was performed to measure the expression of miR-18a-5p and CASC2. mRNA expression of CASC2 (D) and miR-18a-5p (E) in NPC cells transfected with si-NC or si-CASC2 was determined using RT-qPCR. "P<0.05 compared with the respective control. CASC2, cancer susceptibility candidate 2; NPC, nasopharyngeal carcinoma; WT, wild-type; MUT, mutant.
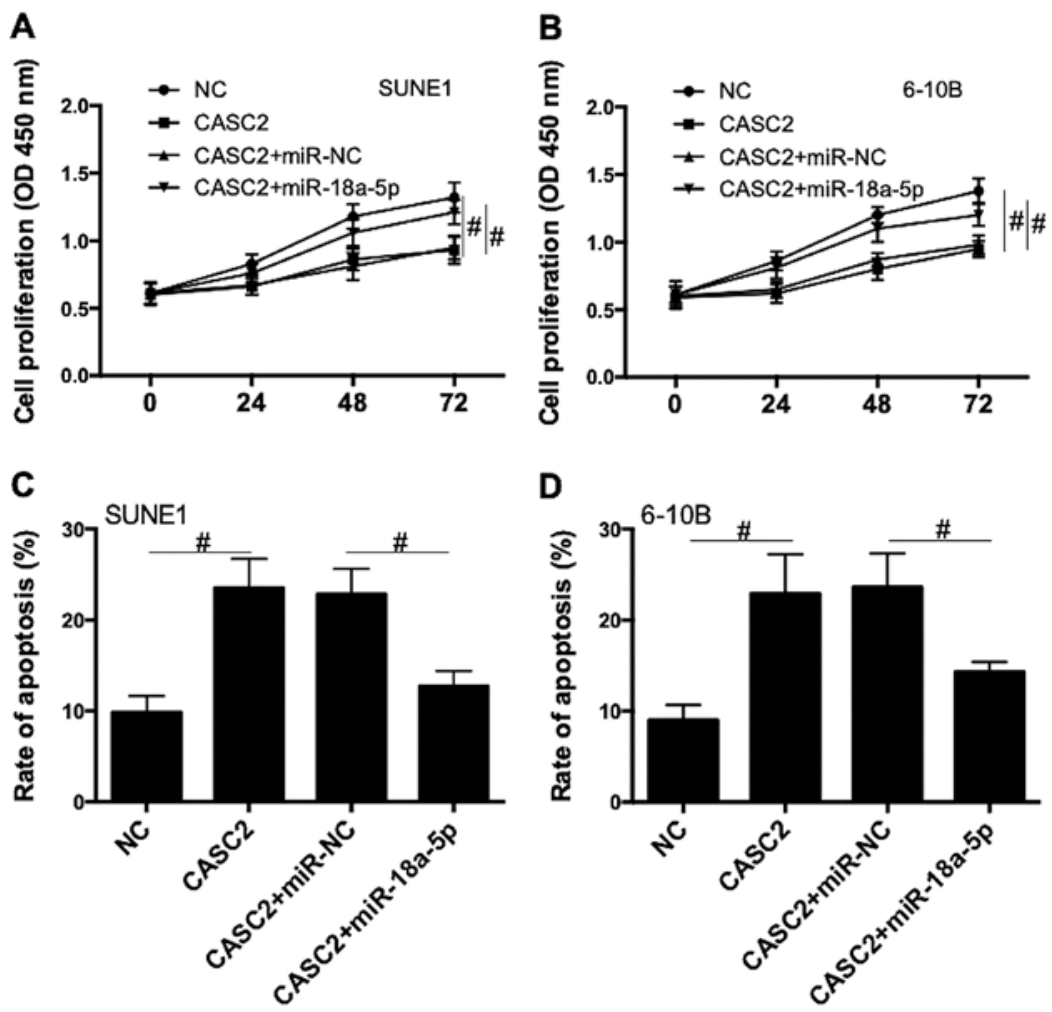

Figure 4. miR-18a-5p mimic suppresses CASC2 upregulation-induced reduction in proliferation and increase in apoptosis in NPC cells. (A and B) Cell proliferation of NPC cells transfected with pcDNA or pcDNA-CASC2 or together with miR-NC or miR-18a-5p was determined using the CCK-8 assay. (C and D) Apoptosis of NPC cells transfected with pcDNA or pcDNA-CASC2 or together with miR-NC or miR-18a-5p was measured via TUNEL assay. ${ }^{\#} \mathrm{P}<0.05$ compared with the respective control. NPC, nasopharyngeal carcinoma; CASC2, cancer susceptibility candidate 2; CCK-8, Cell Counting Kit-8. 
A

RBBP8 WT

$\operatorname{miR}-18 a-5 p$

RBBP8 MUT
5'-ugauauuuugcuuuuGCACCUUu-3'

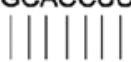

3'-gauugacgugaucuaCGUGGAAu5'

5'-ugauauuuugcuuuuAUGUUCCu-3'
B

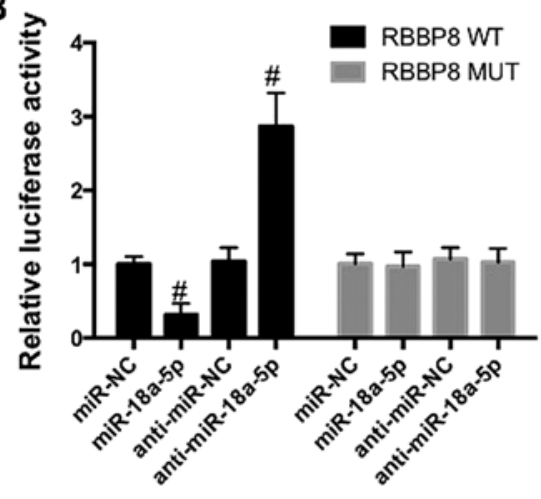

C

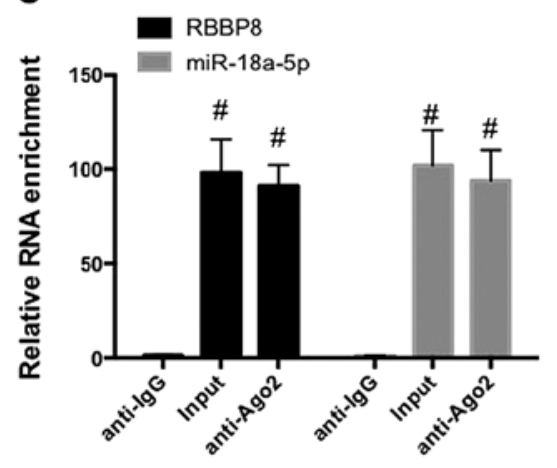

D

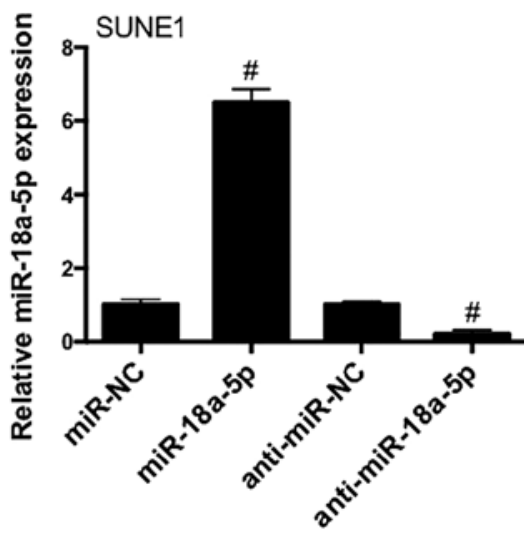

E

Figure 5. miR-18a-5p suppresses RBBP8 expression by direct interaction. (A) Bioinformatic analysis was performed to find putative binding sites between RBBP8 and miR-18a-5p. (B) 293T cells were co-transfected with WT or MUT RBBP8 luciferase vectors and anti-miR-NC or anti-miR-18a-5p and miR-NC or miR-18a-5p, and then the direct interaction between CASC2 and miR-18a-5p was assessed by reporter gene assay. (C) Cell lysate of SUNE1 cells was incubated with anti-Ago2 antibody or anti-IgG antibody to purify RNA complex and RT-qPCR was performed to measure the expression of miR-18a-5p and RBBP8. mRNA expression of RBBP8 (D) and miR-18a-5p (E) in anti-miR-NC or anti-miR-18a-5p-transfected NPC cells was determined using RT-qPCR. ${ }^{\# P}<0.05$ compared with the respective control. CASC2, cancer susceptibility candidate 2; WT, wild-type; MUT, mutant.

The data suggest that CASC2 acts as a tumor suppressor by acting as ceRNA of miR-18a-5p in NPC cells.

miR-18a-5p promotes cell proliferation and inhibits apoptosis by the regulation of $R B B P 8$. To elucidate the underlying mechanism of miR-18a-5p-induced regulation of NPC progression, we aimed to identify possible targets of miR-18a-5p using bioinformatic analysis. The results revealed that RBBP8 possesses complementary sites of miR-18a-5p (Fig. 5A), indicating that there is a possible interaction between miR-18a-5p and RBBP8. 293T cells were co-transfected with constructed WT and MUT RBBP8 luciferase vectors with or without anti-miR-NC or anti-miR-18a-5p and miR-NC or miR-18a-5p mimics. The results revealed that miR-18a-5p mimics significantly decreased the luciferase activities of RBBP8 (WT), while miR-18a-5p inhibitors exhibited an opposite effect (Fig. 5B). However, neither miR-18a-5p mimics nor its inhibitors affected MUT RBBP8 reporter luciferase activities (Fig. 5B). As shown in Fig. 5C, RBBP8 and miR-18a-5p mRNA level were significantly increased in the Ago2 antibody complex in SUNE1 cells. To assess the role of miR-18a-5p in the regulation of RBBP8, SUNE1 cells were transfected with miR-18a-5p mimics or inhibitors (Fig. 5D). miR-18a-5p mimics significantly decreased
RBBP8 expression in SUNE1 cells, while miR-18a-5p inhibitors promoted RBBP8 expression (Fig. 5E). These data demonstrated that RBBP8 may be a target of miR-18a-5p in NPC cells.

Next, SUNE1 and 6-10B cells were transfected with miR-18a-5p inhibitors together with LV-shNC or LV-shRBBP8. miR-18a-5p inhibitors decreased cell proliferation, and this effect was inhibited by RBBP8 knockdown (Fig. 6A and B). In addition, inhibition of miR-18a-5p significantly increased apoptosis and RBBP8 knockdown suppressed this effect (Fig. 6C and D). The results indicated that RBBP8 functions as a target of miR-18a-5p in NPC cells.

CASC2 knockdown promotes tumor growth, promotes miR-18a-5p expression and inhibits RBBP8 expression in vivo. To further examined the role of CASC2 in vivo, tumor growth in xenograft mice was investigated. CASC2 knockdown significantly promoted the growth of tumors, as illustrated by the increase in tumor volume (Fig. 7A) and tumor weight (Fig. 7B and C). Additionally, CASC2 knockdown (Fig. 7D) increased miR-18a-5p expression (Fig. 7E) and decreased RBBP8 expression (Fig. 7F) in tumor tissues. These results revealed that CASC 2 knockdown promoted tumor growth, promoted miR-18a-5p expression and inhibited RBBP8 expression in vivo. 

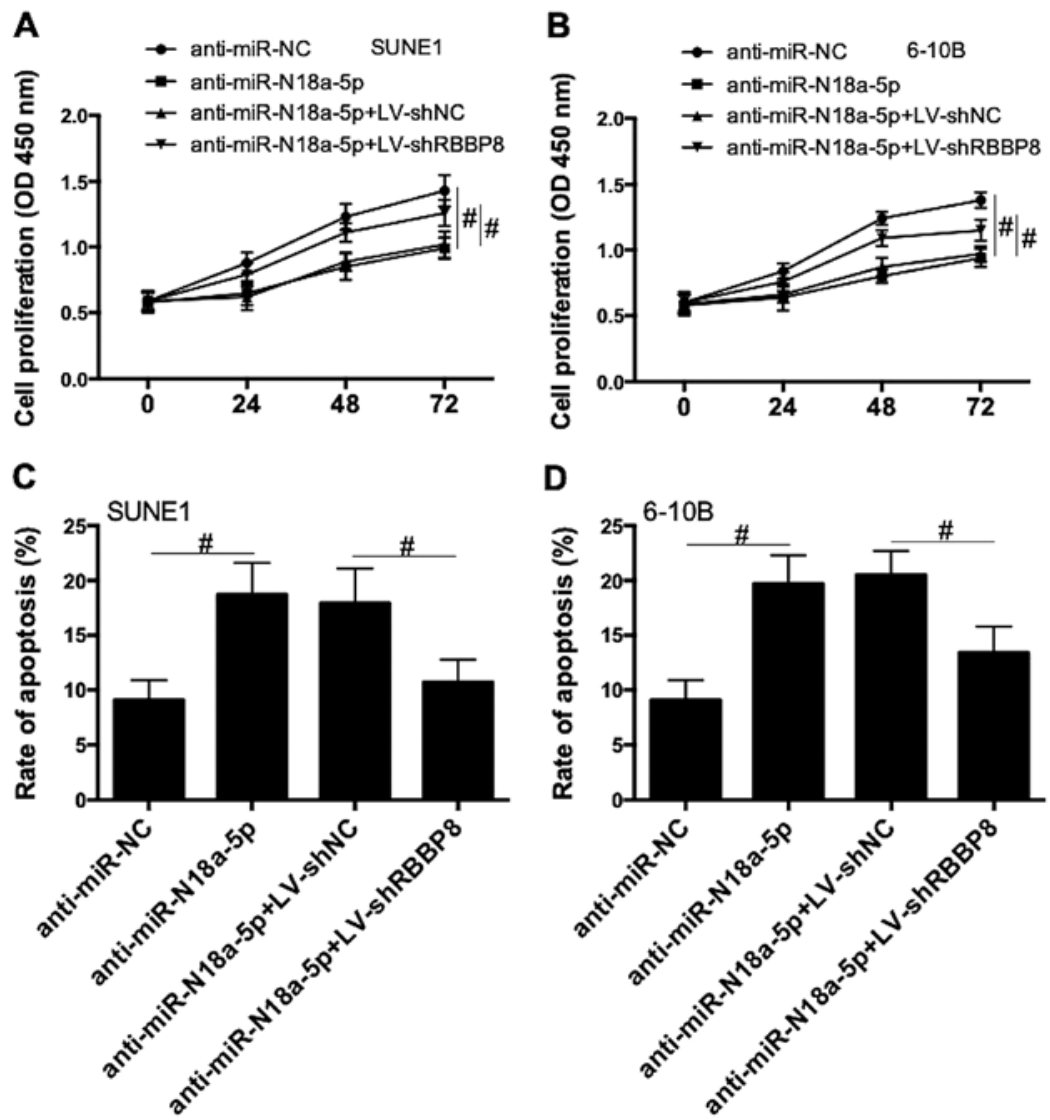

Figure 6. Downregulation of RBBP8 inhibits miR-18a-5p inhibitor-induced decrease in proliferation and increase in apoptosis in NPC cells. (A and B) Cell proliferation of NPC cells transfected with anti-miR-NC or anti-miR-18a-5p or together with LV-shNC or LV-shRBBP8 was determined using the CCK-8 assay. (C and D) Apoptosis of NPC cells transfected with anti-miR-NC or anti-miR-18a-5p or together with LV-shNC or LV-shRBBP8 was determined using TUNEL assay. ${ }^{*} \mathrm{P}<0.05$ compared with the respective control. NPC, nasopharyngeal carcinoma; CCK-8, Cell Counting Kit-8.

\section{Discussion}

Cancer susceptibility candidate 2 (CASC2), a newly identified lncRNA, is located on chromosome 10q26 (30). It was initially found that CASC2 expression is reduced in endometrial carcinoma and downregulation of CASC2 provides a growth advantage in endometrial cancer cells $(30,31)$. Subsequently, CASC2 was found to play a tumor suppressive role in several human types of cancers, including hepatocellular carcinoma, bladder, breast cancer, osteosarcoma, gastric cancer, lung adenocarcinoma and endometrial cancer (19-25). We revealed, in the present study, a significant decrease in CASC2 expression in nasopharyngeal carcinoma (NPC) tissues and cells (Fig. 1). Moreover, downregulation of CASC2 promoted proliferation and inhibited apoptotic cell death in SUNE1 and 6-10B cells, while CASC2 upregulation inhibited cell proliferation and promoted apoptosis (Fig. 2). The tumor-suppressive role in vivo was also confirmed in xenograft mice (Fig. 7). The data suggested that CASC2 may be a key regulator in the tumorigenesis of NPC.

The regulatory mechanisms of lncRNAs include signals, decoys, guides and scaffolds (32). As a decoy, lncRNAs function via binding microRNAs or proteins to modulate the functions of key molecules (33). Numerous investigations have suggested that CASC 2 may function to sponge miRNAs, which leads to the inhibition of miRNA-induced regulation of target mRNAs and thus the regulation of many cancers $(20,22-24,34)$. For example, CASC2 downregulation was found to promote the growth and invasion of osteosarcoma through regulation of miR-181a (22). In hepatocellular carcinoma, CASC2 regulates miR-24-3p, leading to a decrease in cell viability and an increase in apoptosis (23). CASC2 was found to modulate docetaxel-induced sensitivity in prostate cancer cells via regulation of miR-183/Sprouty 2 signaling (34).

MicroRNAs are a class of short (18-24 nt), single stranded and non-coding RNAs. These RNAs could directly bind with the target mRNAs, leading to transcriptional regulation (35). A battery of studies have shown that dysfunction of miR-18a-5p is associated with cancer pathogenesis. Recently, it was reported that GAS5 could regulate miR-18a-5p and thus modulate proliferation, migration and invasion in glioma cells (36). In breast cancer, miR-18a-5p could target SREBP1 and modulate epithelial-mesenchymal transition (37). miR-18a-5p was also reported to function as an oncogene and prognostic biomarker in the development of RCC (38). In lung cancer, miR-18a-5p plays an oncogenic role via direct regulation of IRF2 (39). In the present study, we revealed that miR-18a-5p is a target of CASC2, and CASC2 suppresses the expression of miR-18a-5p. miR-18a-5p expression was significantly increased in NPC tissues and cells (Fig. 1). miR-18a-5p mimics inhibited CASC2 overexpression-induced reduction in proliferation and increase in apoptosis (Fig. 3). The data suggest that miR-18a-5p promoted NPC tumorigenesis. 

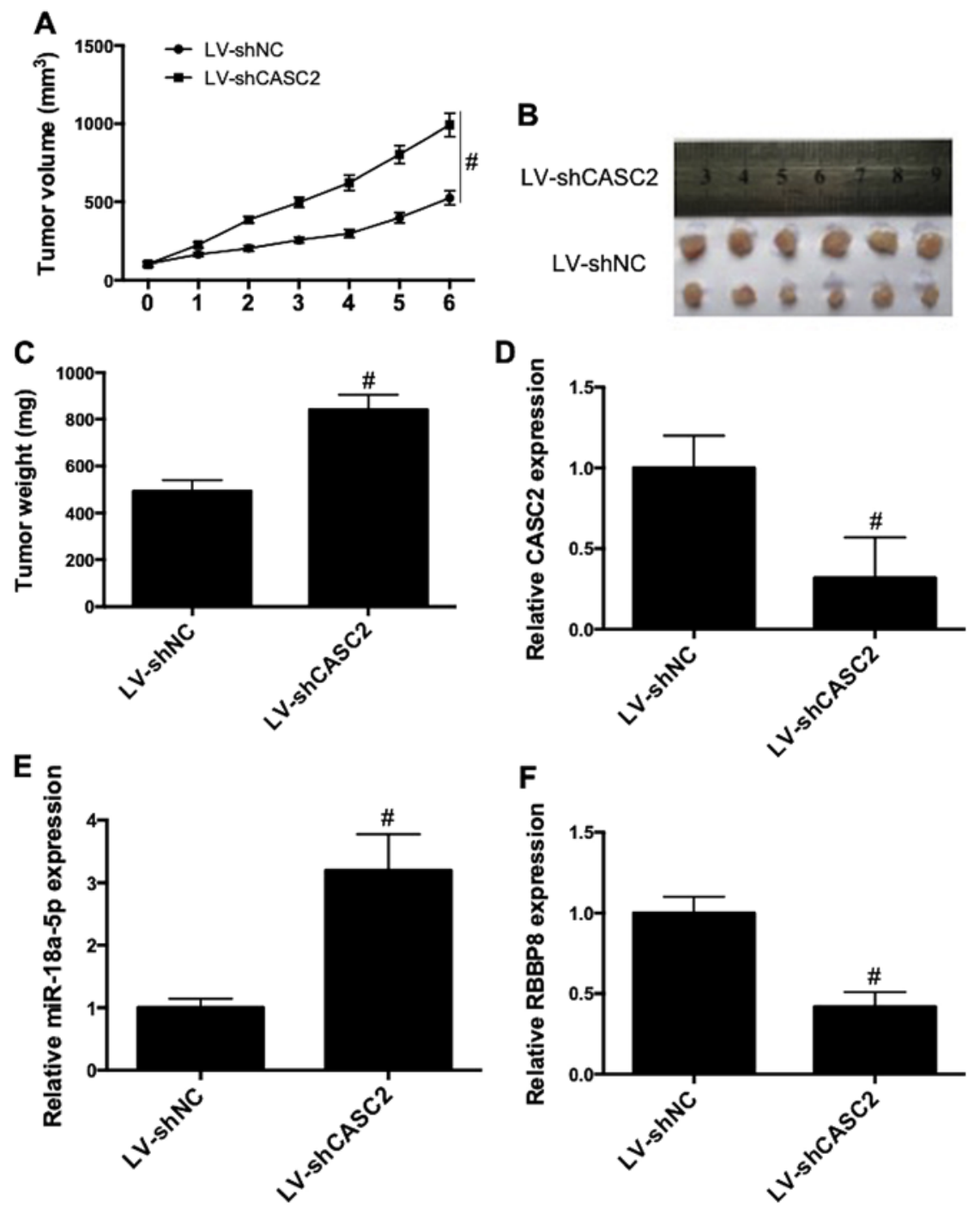

Figure 7. CASC2 knockdown promotes tumor growth, promotes miR-18a-5p expression, and inhibits RBBP8 expression in vivo. Approximately $8 \times 10^{6}$ SUNE 1 cells with or without stable knockdown of CASC2 were subcutaneously inoculated into the mice to establish a tumor-transplantion model. (A) The volume of the tumors was determined using a caliper. (B) Tumor images isolated from the transplanted mice. (C) Tumor weight was measured after the sacrifice of mice. (D) mRNA expression of CASC2 in tumors was determined using RT-qPCR. (E) mRNA expression of miR-18a-5p in tumors was determined. (F) RBBP8 mRNA expression in tumors was determined. ${ }^{\#} \mathrm{P}<0.05$ compared with the respective control. CASC2, cancer susceptibility candidate 2 .

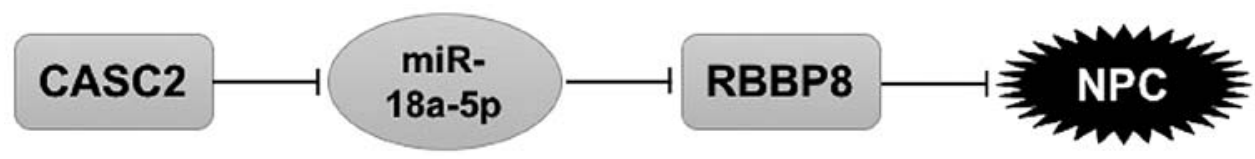

Figure 8. The CASC2/miR-18a-5p/RBBP8 axis in the regulation of NPC. The findings in the present study suggest that CASC2 plays a tumor-suppressive role in NPC and downregulation of CASC2 is critical for cell proliferation and tumor growth. CASC2 regulates the malignant potential of NPC through modulation of RBBP8 via sponging miR-18a-5p. CASC2, cancer susceptibility candidate 2; NPC, nasopharyngeal carcinoma.

The possible underlying mechanisms of the oncogenic role of miR-18a-5p in NPC were investigated. Bioinformatic analysis suggested the possible interaction between miR-18a-5p and RBBP8 (Fig. 5). It was previously described that CtIP/RBBP8 is a transcriptional corepressor (40), which was shown to bind several other transcription factors such as TRB3, LMO4 and Ikaros that are associated with cancer $(41,42)$. Frameshift mutations induced by CtIP/RBBP8 microsatellite have been found in colorectal (43) and endometrial cancer (44). From a mechanistic point, CtIP/RBBP8 regulates the cell cycle by interacting with RB1 (45). In breast cancer, a reduction in CtIP/RBBP8 expression is associated with an increase in disease-free survival under the condition of co-treatment of hormone, radiotherapy and chemotherapy (46). In the present study, the results suggested that miR-18a-5p targeted RBBP8 and suppressed its expression (Fig. 5). RBBP8 knockdown blocked the decrease in cell proliferation and increase in apoptosis induced by miR-18a-5p inhibitors (Fig. 6).

In summary, the present study identified that downregulation of CASC2 is critical for the cell proliferation and tumor 
growth of NPC. CASC2 regulates the malignant potential of NPC through modulation of RBBP8 via sponging miR-18a-5p (Fig. 8). Our findings highlight the CASC2/miR-18a-5p/RBBP8 axis in NPC pathogenesis and provide new biomarkers and potential targets for the treatment of NPC.

\section{Acknowledgements}

Not applicable.

\section{Funding}

No funding was received.

\section{Availability of data and materials}

Data will be avaible on request.

\section{Authors' contributions}

WJM and QQJ conceived and designed the study. QQJ, DJY, GZZ, QL and HMM performed the experiments and analyzed the data. WJM, QQJ and DJY prepared the figures. WJM and QQJ wrote the manuscript. All authors read and approved the manuscript and agree to be accountable for all aspects of the research in ensuring that the accuracy or integrity of any part of the work are appropriately investigated and resolved.

\section{Ethics approval and consent to participate}

The present study was conducted according to the principles expressed in the Declaration of Helsinki, and the use of clinical sample tissues was approved by the Ethics Committee of the First Affiliated Hospital of Xinxiang Medical University (Weihui, China). The animal experiment was carried out following the Guidelines for the Care and Use of Laboratory Animals of the National Institutes of Health. The study was approved by the Ethics Committee of the First Affiliated Hospital of Xinxiang Medical University. Written informed consent was obtained from all the enrolled patients.

\section{Patient consent for publication}

Not applicable.

\section{Competing interests}

The authors declare that they have no competing interests.

\section{References}

1. Chen $\mathrm{W}$ and $\mathrm{Hu} \mathrm{GH}$ : Biomarkers for enhancing the radiosensitivity of nasopharyngeal carcinoma. Cancer Biol Med 12: 23-32, 2015.

2. Vokes EE,Liebowitz DN and Weichselbaum RR: Nasopharyngeal carcinoma. Lancet 350: 1087-1091, 1997.

3. Petersson F: Nasopharyngeal carcinoma: A review. Semin Diagn Pathol 32: 54-73, 2015.

4. Zhou X, Xiao X, Huang T, Du C, Wang S, Mo Y, Ma N, Murata M, Li B, Wen W, et al: Epigenetic inactivation of follistatin-like 1 mediates tumor immune evasion in nasopharyngeal carcinoma. Oncotarget 7: 16433-16444, 2016.
5. Aldape K, Zadeh G, Mansouri S, Reifenberger G and von Deimling A: Glioblastoma: Pathology, molecular mechanisms and markers. Acta Neuropathol 129: 829-848, 2015.

6. Shi W, Bastianutto C, Li A, Perez-Ordonez B, Ng R, Chow KY, Zhang W, Jurisica I, Lo KW, Bayley A, et al: Multiple dysregulated pathways in nasopharyngeal carcinoma revealed by gene expression profiling. Int J Cancer 119: 2467-2475, 2006.

7. Altun M, Fandi A, Dupuis O, Cvitkovic E, Krajina Z and Eschwege F: Undifferentiated nasopharyngeal cancer (UCNT): Current diagnostic and therapeutic aspects. Int J Radiat Oncol Biol Phys 32: 859-877, 1995.

8. Tan WL, Tan EH, Lim DW, Ng QS, Tan DS, Jain A and Ang MK: Advances in systemic treatment for nasopharyngeal carcinoma. Chin Clin Oncol 5: 21, 2016.

9. Parkin DM and Muir CS: Cancer incidence in five continents. Comparability and quality of data. IARC Sci Publ 120: 45-173, 1992.

10. Lee AW, Sze WM, Au JS, Leung SF, Leung TW, Chua DT, Zee BC, Law SC, Teo PM, Tung SY, et al: Treatment results for nasopharyngeal carcinoma in the modern era: The Hong Kong experience. Int J Radiat Oncol Biol Phys 61: 1107-1116, 2005.

11. Cai X, Liu Y, Yang W, Xia Y, Yang C, Yang S and Liu X: Long noncoding RNA MALAT1 as a potential therapeutic target in osteosarcoma. J Orthop Res 34: 932-941, 2016.

12. Gao KT and Lian D: Long non-coding RNA MALAT1 is an independent prognostic factor of osteosarcoma. Eur Rev Med Pharmacol Sci 20: 3561-3565, 2016.

13. Huo Y, Li Q, Wang X, Jiao X, Zheng J, Li Z and Pan X: MALAT1 predicts poor survival in osteosarcoma patients and promotes cell metastasis through associating with EZH2. Oncotarget 8: 46993-47006, 2017.

14. Li Q, Pan X, Wang X, Jiao X, Zheng J, Li Z and Huo Y: Long noncoding RNA MALAT1 promotes cell proliferation through suppressing miR-205 and promoting SMAD4 expression in osteosarcoma. Oncotarget 8: 106648-106660, 2017.

15. Fan L, Huang C, Li J, Gao T, Lin Z and Yao T: Long non-coding RNA urothelial cancer associated 1 regulates radioresistance via the hexokinase $2 /$ glycolytic pathway in cervical cancer. Int J Mol Med 42: 2247-2259, 2018.

16. Wu X, Tudoran OM, Calin GA and Ivan M: The many faces of long noncoding RNAs in cancer. Antioxid Redox Signal 29: 922-935, 2018.

17. Zhang H, Cai Y, Zheng L, Zhang Z, Lin X and Jiang N: Long noncoding RNA NEAT1 regulate papillary thyroid cancer progression by modulating miR-129-5p/KLK7 expression. J Cell Physiol 233: 6638-6648, 2018.

18. Martens-Uzunova ES, Bottcher R, Croce CM, Jenster G, Visakorpi $\mathrm{T}$ and Calin GA: Long noncoding RNA in prostate, bladder, and kidney cancer. Eur Urol 65: 1140-1151, 2014.

19. Zhang Y, Zhu M, Sun Y, Li W, Wang Y and Yu W: Up-regulation of lncRNA CASC2 suppresses cell proliferation and metastasis of breast cancer via inactivating of the TGF- $\beta$ signaling pathway. Oncol Res: Mar 9, 2018 (Epub ahead of print). doi: 10.3727/0965 04018X15199531937158.

20. Zhao L and Zhang Y: Long noncoding RNA CASC2 regulates hepatocellular carcinoma cell oncogenesis through miR-362-5p/Nf- $\mathrm{kB}$ axis. J Cell Physiol 233: 6661-6670, 2018.

21. Zhou J, Huang H, Tong S and Huo R: Overexpression of long non-coding RNA cancer susceptibility 2 inhibits cell invasion and angiogenesis in gastric cancer. Mol Med Rep 16: 5235-5240, 2017.

22. Ba Z, Gu L, Hao S, Wang X, Cheng Z and Nie G: Downregulation of lncRNA CASC2 facilitates osteosarcoma growth and invasion through miR-181a. Cell Prolif 51: e12409, 2018.

23. Fan JC, Zeng F, Le YG and Xin L: IncRNA CASC2 inhibited the viability and induced the apoptosis of hepatocellular carcinoma cells through regulating miR-24-3p. J Cell Biochem 119: 6391-6397, 2018

24. LiH,ShiH,GaoM,MaNandSun R:Longnon-coding RNACASC2 improved acute lung injury by regulating miR-144-3p/AQP1 axis to reduce lung epithelial cell apoptosis. Cell Biosci 8: 15, 2018.

25. Wang D, Gao ZM, Han LG, Xu F, Liu K and Shen Y: Long noncoding RNA CASC2 inhibits metastasis and epithelial to mesenchymal transition of lung adenocarcinoma via suppressing SOX4. Eur Rev Med Pharmacol Sci 21: 4584-4590, 2017.

26. Calin GA and Croce CM: Chromosomal rearrangements and microRNAs: A new cancer link with clinical implications. J Clin Invest 117: 2059-2066, 2007.

27. Liz $\mathbf{J}$ and Esteller M: lncRNAs and microRNAs with a role in cancer development. Biochim Biophys Acta 1859: 169-176, 2016. 
28. Yoon JH, Abdelmohsen $\mathrm{K}$ and Gorospe M: Functional interactions among microRNAs and long noncoding RNAs. Semin Cell Dev Biol 34: 9-14, 2014

29. Livak KJ and Schmittgen TD: Analysis of relative gene expression data using real-time quantitative PCR and the 2(-Delta Delta C(T)) method. Methods 25: 402-408, 2001.

30. Baldinu P, Cossu A, Manca A, Satta MP, Sini MC, Rozzo C, Dessole S, Cherchi P, Gianfrancesco F, Pintus A, et al: Identification of a novel candidate gene, CASC2, in a region of common allelic loss at chromosome 10q26 in human endometrial cancer. Hum Mutat 23: 318-326, 2004.

31. Baldinu P, Cossu A, Manca A, Satta MP, Sini MC, Palomba G, Dessole S, Cherchi P, Mara L, Tanda F and Palmieri G: CASC2a gene is down-regulated in endometrial cancer. Anticancer Res 27: 235-243, 2007.

32. Wang $\mathrm{KC}$ and Chang HY: Molecular mechanisms of long noncoding RNAs. Mol Cell 43: 904-914, 2011.

33. Qu L, Ding J, Chen C, Wu ZJ, Liu B, Gao Y, Chen W, Liu F, Sun W, Li XF, et al: Exosome-transmitted lncARSR promotes sunitinib resistance in renal cancer by acting as a competing endogenous RNA. Cancer Cell 29: 653-668, 2016.

34. Gao W, Lin S, Cheng C, Zhu A, Hu Y, Shi Z, Zhang X and Hong Z: Long non-coding RNA CASC2 regulates Sprouty2 via functioning as a competing endogenous RNA for miR-183 to modulate the sensitivity of prostate cancer cells to docetaxel Arch Biochem Biophys. 2018 Jan 23. pii: S0003-9861(17)30840-8. doi: 10.1016/j.abb.2018.01.013.

35. Iorio MV and Croce CM: MicroRNA dysregulation in cancer: Diagnostics, monitoring and therapeutics. A comprehensive review. EMBO Mol Med 9: 852, 2017.

36. Liu Q, Yu W, Zhu S, Cheng K, Xu H, Lv Y, Long X, Ma L, Huang J, Sun S and Wang K: Long noncoding RNA GAS5 regulates the proliferation, migration, and invasion of glioma cells by negatively regulating miR-18a-5p. J Cell Physiol 234: 775-768, 2018.

37. Zhang N, Zhang H, Liu Y, Su P, Zhang J, Wang X, Sun M, Chen B, Zhao W, Wang L, et al: SREBP1, targeted by miR-18a-5p, modulates epithelial-mesenchymal transition in breast cancer via forming a co-repressor complex with snail and HDAC1/2. Cell Death Differ: Jul 9, 2018 (Epub ahead of print). doi: 10.1038/ s41418-018-0158-8.
38. Zhou L, Li Z, Pan X, Lai Y, Quan J, Zhao L, Xu J, Xu W, Guan X, $\mathrm{Li} \mathrm{H}$, et al: Identification of miR-18a-5p as an oncogene and prognostic biomarker in RCC. Am J Transl Res 10: 1874-1886, 2018.

39. Liang C, Zhang X, Wang HM, Liu XM, Zhang XJ, Zheng B, Qian GR and Ma ZL: MicroRNA-18a-5p functions as an oncogene by directly targeting IRF2 in lung cancer. Cell Death Dis 8: e2764, 2017.

40. Schaeper U, Subramanian T, Lim L, Boyd JM and Chinnadurai G: Interaction between a cellular protein that binds to the $\mathrm{C}$-terminal region of adenovirus E1A (CtBP) and a novel cellular protein is disrupted by E1A through a conserved PLDLS motif. J Biol Chem 273: 8549-8552, 1998.

41. Koipally J and Georgopoulos K: Ikaros-CtIP interactions do not require $\mathrm{C}$-terminal binding protein and participate in a deacetylase-independent mode of repression. J Biol Chem 277: 23143-23149, 2002.

42. Sum EY, Peng B, Yu X, Chen J, Byrne J, Lindeman GJ and Visvader JE: The LIM domain protein LMO4 interacts with the cofactor CtIP and the tumor suppressor BRCA1 and inhibits BRCA1 activity. J Biol Chem 277: 7849-7856, 2002.

43. Ikenoue T, Togo G, Nagai K, Ijichi H, Kato J, Yamaji Y, Okamoto M, Kato N, Kawabe T, Tanaka A, et al: Frameshift mutations at mononucleotide repeats in RAD50 recombinational DNA repair gene in colorectal cancers with microsatellite instability. Jpn J Cancer Res 92: 587-591, 2001.

44. Bilbao C, Ramirez R, Rodriguez G, Falcón O, León L, Díaz-Chico N, Perucho M and Díaz-Chico JC: Double strand break repair components are frequent targets of microsatellite instability in endometrial cancer. Eur J Cancer 46: 2821-2827, 2010.

45. Fusco C, Reymond A and Zervos AS: Molecular cloning and characterization of a novel retinoblastoma-binding protein. Genomics 51: 351-358, 1998.

46. Yamaguchi N, Osaki M, Onuma K, Yumioka T, Iwamoto $\mathrm{H}$, Sejima T, Kugoh H, Takenaka A and Okada F: Identification of microRNAs involved in resistance to sunitinib in renal cell carcinoma cells. Anticancer Res 37: 2985-2992, 2017. 